\title{
Effect of Media on Root Parameters in Marigold
}

\author{
J. Vijay ${ }^{1}$, A.V.D. Dorajeerao ${ }^{2}$, K. Umajyothi $^{3}$ and D.R. Salomi Suneetha ${ }^{4}$ \\ ${ }^{1}$ College of Horticulture, Dr. Y.S.R. Horticultural University, V.R. Gudem-534101, \\ Andhra Pradesh, India \\ 2(Floriculture and Landscape Architecture), College of Horticulture, Dr. Y.S.R. Horticultural \\ University, V.R. Gudem-534101, Andhra Pradesh, \\ 3(Horticulture), College of Horticulture, 4(Biochemistry), College of Horticulture, Dr. Y.S.R. \\ Horticultural University, V.R. Gudem-534101, Andhra Pradesh, India \\ *Corresponding author
}

\section{A B S T R A C T}

Keywords

Rooted cuttings, Roots, Fresh weight, Dryweight

Article Info

Accepted:

26 October 2018

Available Online:

10 November 2018
An experiment was carried in factorial concept with two factors i.e., varieties at two levels and rooting media at eight levels thus making 16 combinations which were replicated twice. The effect of varieties, rooting media and their interactions were found to be significant on different rooting parameters. Among the rooting media, the greatest percentage of rooted cuttings was recorded by M8 - soil:vermicompost : coco peat : saw dust $(1: 1: 1: 1) \mathrm{v} / \mathrm{v}+$ mycorrhiza. At 90 days after shifting into polybags, the number of roots per cutting, length of the longest root per cutting, fresh weight of roots per cutting, maximum survival percentage of rooted cuttings were found to be superior with the media M8 - soil : vermicompost : coco peat : saw dust $(1: 1: 1: 1) \mathrm{v} / \mathrm{v}+$ mycorrhiza whereas dry weight of roots per cutting were found to be superior with the media M6 -soil : vermicompost : coco peat : rice husk $(1: 1: 1: 1) \mathrm{v} / \mathrm{v}+$ mycorrhiza.

\section{Introduction}

Marigold (Tagetes erecta L.) is one of the most important commercial flower crops grown in India. The crop is said to be native of Mexico. It belongs to the family Asteraceae and propagated by seed and terminal cuttings.

A higher number of roots could be seen in terminal cuttings of marigold than in seed propagation of marigold, helps to increase number of branches, maximum leaves per plant, maximum plant height, large flower bloom than those plants propagated sexually through seed. Rooting media are essential for improvement in rooting percentage and hence the selection and combination of media components assumes greater significance for success in vegetative propagation (Laubscher, 1990).

Moisture holding capacity and optimum aeration have been regarded as the most essential characteristics for rooting medium. The medium used for rooting of cuttings should be also firm so as to hold the plant or cutting in position. 
Therefore an ideal rooting medium should be porous enough to allow good aeration and should possess high water holding ability.

The type of rooting medium determines to some extent, the nature of roots which are produced on the cutting and consequently its survival. Thus medium for rooting has gained lot of importance in propagation of marigold. The rooting media has been reported to influence the success of propagation in several crops through vegetative parts.

\section{Material and Methods}

Experiment was carried out at College farm, College of Horticulture, Dr. Y.S.R. Horticultural University, Venkataramanna gudem, West Godavari District of Andhra Pradesh during rabi, 2017-2018. Experiment was laid out in completely randomised design with factorial concept. Factor one (V) consisted of two levels one being Bidhan Marigold- 1 (V1) and another Bidhan Marigold- 2(V2) and factor two consists of 8 levels of rooting media M1 - soil, M2 - soil + mycorrhiza, M3 - soil: vermicompost (1:1) v/v + mycorrhiza, M4 - soil: vermicompost: coco peat (1:1:1) v/v + mycorrhiza, M5 - soil: vermicompost: rice husk (1:1:1) v/v + mycorrhiza, M6- soil: vermicompost: coco peat: rice husk (1:1:1:1) v/v + mycorrhiza, M7 - soil: vermicompost: saw dust (1:1:1) v/v + mycorrhiza and M8- soil: vermicompost: coco peat: saw dust (1:1:1:1) v/v + mycorrhiza. Thus, there were 16 treatment combinations and were repeated twice. Mycorrhiza was applied at the rate of $2 \mathrm{~g}$ per plant (Neelima $e t$ al., 2016). The following observations on root parameters were recorded at regular interval starting from the time of shifting and at 30,60 and 90 days after shifting (DAS) of the cuttings. Percentage of rooted cuttings (\%): This parameter was recorded at the time of shifting the rooted cuttings from shade house by using the following formula.
Percentage of rooted cuttings $(\%)=[$ No of cuttings rooted / Total no of cuttings planted] X 100

Number of roots per cutting, Length of longest root $(\mathrm{cm})$, Fresh weight of roots/cutting, Dry weight of roots/cutting, Survival \% of rooted cuttings. The experimental data pertaining to all the characters studied were subjected to statistical analysis of variance technique as described by Panse and Sukhatme (1997).

\section{Results and Discussion}

\section{Percentage of rooted cuttings (\%) (Table 1)}

Among the rooting media, the greatest percentage of rooted cuttings $(93.35 \%)$ was recorded by M8 - soil: vermicompost: coco peat: saw dust (1:1:1:1) v/v + mycorrhiza which was on par with M6 - soil: vermicompost: coco peat: rice husk (1:1:1:1) $\mathrm{v} / \mathrm{v}+$ mycorrhiza $(92.95 \%)$ and M4 - soil: vermicompost: coco peat $(1: 1: 1) \mathrm{v} / \mathrm{v}+$ mycorrhiza $(92.95 \%)$ but significantly superior to M3 - soil: vermicompost (1:1) v/v + mycorrhiza (92.13\%). The lowest percentage of rooted cuttings $(88.78 \%)$ was recorded on M1 - soil.

Among the rooting media, terminal cuttings planted in coco peat based media recorded the highest percentage of rooting which might be perhaps due to the release of phenolic compounds from the coir pith (Lokesha et al., 1988) and also can be attributed to the beneficial physical characteristics of coir pith (Smith, 1995) like aeration and water holding capacity. Presence of tender leaves on cuttings also could have played an important role in the initiation of roots in many plant species. Leaves considerably influence the rooting of cuttings because of their ability to produce endogenous auxins, carbohydrates by means of photosynthesis (Newton et al., 1992). In addition, vermicompost could have provided 
nutrients and stabilized the $\mathrm{pH}$ due to humus content in the rooting media. These characteristics of cocopeat and vermicompost might have played a synergist and important role in increasing percentage of rooted cuttings. Similarly, Shirol et al., (2001) also reported the highest percentage of rooted cuttings of dwarf poinsettia in media containing vermicompost + sand (1:1) medium (67\%), followed by vermicompost alone. Ochoa et al., (2003) obtained highest rooting of oleander cuttings in cocopeat.

Mycorrhizal fungi are important to their hosts as they enhance the ability of plants to absorb phosphorus from soil, which is relatively unavailable to the plants (Mcgonigle and Miller, 1996; Miller, 2000). They secrete organic acids viz., formic, acetic, propionic, lactic, glycolic, fumaric and succinic acids, vitamins and growth promoting substances like IAA and gibberellins which helps in better plant growth (Reddy, 2008). This could be the reason as to why the mycorhizal combinations were better than non-mycorhizal combinations of rooting media.

\section{Number of roots per cutting (Table 2)}

Among the rooting media, at 90 DAS the highest number of roots (157.85) was recorded by M8 - soil: vermicompost: coco peat: saw dust $(1: 1: 1: 1) \mathrm{v} / \mathrm{v}+$ mycorrhiza followed by M6 - soil: vermicompost: coco peat: rice husk $(1: 1: 1: 1) \mathrm{v} / \mathrm{v}+$ mycorrhiza (135.15) and M4 soil: vermicompost: coco peat $(1: 1: 1) \mathrm{v} / \mathrm{v}+$ mycorrhiza (126.30). The lowest number of roots (67.83) was recorded on M1 - soil. The interactions with Bidhan Marigold 1 were found to show superior values when compared to those with the variety Bidhan Marigold 2.

The terminal cuttings planted in coco peat medium gave more number of roots, which might be due to the presence of cytokinins in coco peat that encouraged the induction of adventitious roots (Ellyard and Ollerenshaw, 1984), along with auxin mediated cell division (Davies et al., 1987) resulting in more number of roots. Coco peat has a high water holding capacity which helps in the absorption of water and nutrients at higher level (Rubasinghe et al., 2009) from the medium thereby increasing the number of roots. The root formation process on the cuttings is a complicated one which is regulated by many different internal factors like the concentration of endogenous auxins, the rooting cofactors, carbohydrate substances stored in the cuttings as well as nitrogen content, these may interact to influence the rooting percentage, root length and diameter and root weight. Cocopeat + vermicompost combinations in the rooting media might had contributed to a better nitrogen content and thereby could have induced more number of roots per cutting. The formation of higher number of roots per cutting may be the fact that the cambial activity is involved in root induction (Rahman et al., 1991).

Such observations were also made by Wahab et al., (2001) in guava, Riaz et al., (2007) in hardwood cuttings of kiwi, Ismail and Asghar (2007) in Ficushawaii, Malik et al., (2013) and Abdul et al., (2013) in guava. Khelwale et al., (2005) reported that coco peat was significantly superior to remaining media treatments in rooting of carnation. Edwards and Neuhauser (1988) reported that vermicompost has maximum pore space, aeration and water holding capacity and has ability to supply nutrients in available form such as nitrate nitrogen and soluble phosphorus, which are necessary for root growth and therefore resulted in maximum number of roots per cutting.

Similar results were reported by Shirol et al., (2001) in dwarf poinsettia, where maximum root development was noticed with vermicomopost + sand $(1: 1)$, followed by 
vermicompost alone. These reports were in confirmation with Mahale et al., (2002) and Bharathy et al., (2003) in carnation. Vesicular arbuscular mycorrhiza is a fungi that colonize the plant root system and develop symbiotic association by forming a network of fine filaments that associate with root system and facilitate increase in absorption of nutrients such as $\mathrm{N}, \mathrm{K}, \mathrm{Mg}$ and $\mathrm{Zn}$ and water from the soil. Plant provides the fungus with photosynthetically derived carbohydrates, while the fungus supplies the plant roots with nutrients (Smith and Read, 2008).

\section{Length of longest root (cm) (Table 3)}

Among the rooting media, at 90 DAS the highest length of longest root $(28.58 \mathrm{~cm})$ was recorded by M8 - soil: vermicompost: coco peat: saw dust (1:1:1:1) v/v + mycorrhiza followed by M6 - soil: vermicompost: coco peat: rice husk (1:1:1:1) v/v + mycorrhiza $(27.50 \mathrm{~cm})$ and M4 - soil: vermicompost: coco peat $(1: 1: 1) \mathrm{v} / \mathrm{v}+$ mycorrhiza $(26.00$ $\mathrm{cm})$. The lowest length of longest root $(16.45$ $\mathrm{cm}$ ) was recorded on M1 - soil.

The possible reason for the longest root per cutting may be due to amount of food reserves in cuttings (Jain et al., 1999).

The terminal cuttings planted in coco peat gave maximum length of root which might be due to better texture and porosity of coco peat, as it enabled the downward movement of water and nutrients (Singh et al., 2002) and lead to easy penetration of roots (Siddagangaiah et al., 1996) in the medium and also being a well-drained one promoting better rooting characters (Singh et al., 2002). Coir has a low particle density indicating its high specific surface that contributes to high adsorption of water and ions by the roots resulting in more root length (Rubasinghe et al., 2009). Norman and Edward (2005) studied the effect of vermicompost on plant growth and reported that vermicompost is a rich source of mineral nutrition and its addition to media increases quality of media by increasing microbial activity and microbial biomass which are key components in nutrient cycling and production of plant growth regulators which might result in superior length of longest root. Shirol et al., (2001) in dwarf poinsettia recorded maximum root development with vermicompost + sand (1:1), followed by vermicompost alone. Cuttings of dwarf poinsettia, highest primary root length was observed in vermicompost medium (Shirol et al., 2001). Further, the association may also increase the phytoavailability of micronutrients, eg., iron, copper, zinc, manganese etc. as the hyphal structures permeate the soil and obtain scarce and relatively immobile elements, by releasing weak phytic acids which break the bonding between soil (positively charges ion) and cationic micro nutrients and makes it available to the plant more effectively than the root hairs of a normal non-mycorrhizal infected plant roots (Smith and Read, 2008). Thus in the present study the positive effect of cocopeat might had been supplemented by that of vermicompost in the media combinations wherever, both of them were used in 1:1 v/v proportion. However, the other components could not make significant difference by their existence in different media combinations so as to deviate from the merit of cocopeat + vermicompost mixtures.

\section{Fresh weight of roots per cutting (g) (Table 4)}

Among the rooting media, at 90 DAS the highest fresh weight of roots (39.43 g) was recorded by M8 - soil: vermicompost: coco peat: saw dust (1:1:1:1) v/v + mycorrhiza followed by M6 - soil: vermicompost: coco peat: rice husk (1:1:1:1) v/v + mycorrhiza (35.38 g) and M4 - soil: vermicompost: coco peat (1:1:1) v/v + mycorrhiza (31.73 g). The lowest fresh weight of roots (20.58 g) was recorded on M1 - soil. 
Table.1 Percentage of rooted cuttings (\%) influenced by rooting media among marigold varieties

\begin{tabular}{|c|c|c|c|}
\hline \multirow[t]{2}{*}{ Variety Medium } & \multicolumn{3}{|c|}{ Percentage of rooted cuttings ( $\%)$} \\
\hline & Bidhan Marigold 1 & Bidhan Marigold 2 & Mean \\
\hline $\mathrm{M}_{1}-$ soil & 90.85 & 86.71 & 88.78 \\
\hline$M_{2}-$ soil + mycorrhiza & 88.35 & 93.35 & 90.85 \\
\hline $\begin{array}{l}M_{3}-\text { soil: vermicompost }(1: 1) \mathrm{v} / \mathrm{v}+ \\
\text { mycorrhiza }\end{array}$ & 92.55 & 91.72 & 92.13 \\
\hline $\begin{array}{l}\mathrm{M}_{4} \text { - soil: vermicompost: coco peat }(1: 1: 1) \mathrm{v} / \mathrm{v} \\
+ \text { mycorrhiza }\end{array}$ & 95.05 & 90.85 & 92.95 \\
\hline $\begin{array}{l}M_{5} \text { - soil: vermicompost: rice husk }(1: 1: 1) \mathrm{v} / \mathrm{v} \\
+ \text { mycorrhiza }\end{array}$ & 93.35 & 90.55 & 91.95 \\
\hline $\begin{array}{l}M_{6}-\text { soil: vermicompost: coco peat: rice husk } \\
(1: 1: 1: 1) \mathrm{v} / \mathrm{v}+\text { mycorrhiza }\end{array}$ & 95.05 & 90.85 & 92.95 \\
\hline $\begin{array}{l}M_{7} \text { - soil: vermicompost: saw dust }(1: 1: 1) \mathrm{v} / \mathrm{v} \\
+ \text { mycorrhiza }\end{array}$ & 91.71 & 92.55 & 92.13 \\
\hline $\begin{array}{l}\text { M8 - soil: vermicompost: coco peat: saw dust } \\
(1: 1: 1: 1) \mathrm{v} / \mathrm{v}+\text { mycorrhiza }\end{array}$ & 95.85 & 90.85 & 93.35 \\
\hline Mean & 92.85 & 91.18 & 92.01 \\
\hline Factor & $\mathrm{S} \mathrm{Em} \pm$ & \multicolumn{2}{|c|}{$\mathrm{CD}$ at $5 \%$} \\
\hline Variety (V) & 0.39 & \multicolumn{2}{|c|}{1.15} \\
\hline Medium (M) & 0.30 & \multicolumn{2}{|c|}{0.89} \\
\hline $\mathbf{V} \times \mathbf{M}$ & 0.59 & \multicolumn{2}{|c|}{1.74} \\
\hline
\end{tabular}


Table.2 Number of roots as influenced by rooting media among marigold varieties

\begin{tabular}{|c|c|c|c|c|c|c|c|c|c|c|c|c|}
\hline \multirow[t]{3}{*}{ VarietyMedium } & \multicolumn{12}{|c|}{ NUMBER OF ROOTS } \\
\hline & \multicolumn{3}{|c|}{ at Shifting } & \multicolumn{3}{|c|}{ at $30 \mathrm{DAS}$} & \multicolumn{3}{|c|}{ at $60 \mathrm{DAS}$} & \multicolumn{3}{|c|}{ at 90 DAS } \\
\hline & $\sum_{n}$ & $\sum_{\infty}^{N}$ & $\underset{\mathbb{E}}{\mathbb{\Xi}}$ & $\sum_{\infty}^{-}$ & $\sum_{n}^{N}$ & $\stackrel{\Xi}{\Xi}$ & $\sum_{\infty}^{-}$ & $\sum_{\infty}^{N}$ & $\stackrel{\Xi}{\Xi}$ & $\sum_{n}^{-}$ & $\sum_{\infty}^{N}$ & 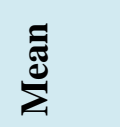 \\
\hline $\mathbf{M}_{1}-$ soil & 21.95 & 50.30 & 36.13 & 37.02 & 23.01 & 30.02 & 60.50 & 48.50 & 54.50 & 80.50 & 55.15 & 67.83 \\
\hline $\mathrm{M}_{2}-$ soil + mycorrhiza & 46.30 & 30.30 & 38.30 & 35.83 & 28.66 & 32.25 & 72.50 & 58.50 & 65.50 & 91.50 & 60.80 & 76.15 \\
\hline $\mathrm{M}_{3}$ - soil: vermicompost $(1: 1) \mathrm{v} / \mathrm{v}+$ mycorrhiza & 50.65 & 29.60 & 40.13 & 43.16 & 43.83 & 43.50 & 66.00 & 75.50 & 70.75 & 76.65 & 94.00 & 85.33 \\
\hline $\begin{array}{l}\mathrm{M}_{4}-\text { soil: vermicompost: coco peat }(1: 1: 1) \mathrm{v} / \mathrm{v}+ \\
\text { mycorrhiza }\end{array}$ & 48.30 & 40.00 & 44.15 & 45.33 & 47.66 & 46.50 & 132.00 & 79.00 & 105.50 & 167.30 & 85.30 & 126.30 \\
\hline $\begin{array}{l}\mathrm{M}_{5}-\text { soil: vermicompost: rice husk }(1: 1: 1) \mathrm{v} / \mathrm{v}+ \\
\text { mycorrhiza }\end{array}$ & 33.95 & 46.65 & 40.30 & 51.33 & 36.33 & 43.83 & 73.00 & 75.00 & 74.00 & 92.30 & 83.30 & 87.80 \\
\hline $\begin{array}{l}\mathrm{M}_{6}-\text { soil: vermicompost: coco peat: rice husk } \\
(1: 1: 1: 1) \mathrm{v} / \mathrm{v}+\text { mycorrhiza }\end{array}$ & 59.95 & 34.50 & 47.23 & 47.49 & 51.83 & 49.66 & 146.00 & 85.00 & 115.50 & 178.00 & 92.30 & 135.15 \\
\hline $\begin{array}{l}\mathrm{M}_{7} \text { - soil: vermicompost: saw dust }(1: 1: 1) \mathrm{v} / \mathrm{v}+ \\
\text { mycorrhiza }\end{array}$ & 45.80 & 39.50 & 42.65 & 40.16 & 49.66 & 44.91 & 106.00 & 66.50 & 86.25 & 143.35 & 76.50 & 109.93 \\
\hline $\begin{array}{l}M_{8} \text { - soil: vermicompost: coco peat: saw dust } \\
(1: 1: 1: 1) \mathrm{v} / \mathrm{v}+\text { mycorrhiza }\end{array}$ & 68.00 & 35.10 & 51.55 & 43.66 & 56.66 & 50.16 & 145.00 & 125.00 & 135.00 & 166.50 & 149.20 & 157.85 \\
\hline Mean & 46.86 & 38.24 & 42.55 & 43.00 & 42.21 & 42.60 & 100.13 & 76.63 & 88.38 & 124.51 & 87.07 & 105.79 \\
\hline Factor & $\mathrm{S} \mathrm{Em}+$ & & at $5 \%$ & $\mathrm{~S} \mathrm{Em}+$ & $\mathrm{CI}$ & at $5 \%$ & $\mathrm{~S} \mathrm{Em} \pm$ & $\mathrm{CD}$ & at $5 \%$ & $\mathrm{~S} \mathrm{Em} \pm$ & $\mathrm{CD}$ & $5 \%$ \\
\hline Variety (V) & 2.03 & 5. & & 0.18 & 0. & & 5.53 & 16 & & 8.82 & 25 . & \\
\hline Medium (M) & 1.00 & 2. & & 1.50 & 4. & & 5.55 & 16. & & 6.35 & 18. & \\
\hline
\end{tabular}


Table.3 Length of longest root as influenced by rooting media among marigold varieties

\begin{tabular}{|c|c|c|c|c|c|c|c|c|c|c|c|c|}
\hline \multirow[t]{3}{*}{ Variety Medium } & \multicolumn{12}{|c|}{ LENGTH OF LONGEST ROOT } \\
\hline & \multicolumn{3}{|c|}{ at Shifting } & \multicolumn{3}{|c|}{ at $30 \mathrm{DAS}$} & \multicolumn{3}{|c|}{ at 60 DAS } & \multicolumn{3}{|c|}{ at 90 DAS } \\
\hline & B M 1 & B M 2 & Mean & $\begin{array}{c}\text { B M } \\
1\end{array}$ & В M 2 & Mean & B M 1 & B M 2 & Mean & B M 1 & B M 2 & Mean \\
\hline$\overline{M_{1}-\text { soil }}$ & 8.35 & 6.30 & 7.33 & 14.89 & 11.51 & 13.20 & 14.90 & 12.00 & 13.45 & 18.30 & 14.60 & 16.45 \\
\hline $\mathbf{M}_{2}$ - soil + mycorrhiza & 8.20 & 7.00 & 7.60 & 18.11 & 13.49 & 15.80 & 12.75 & 14.25 & 13.50 & 16.94 & 23.60 & 20.27 \\
\hline $\begin{array}{l}\mathbf{M}_{3}-\text { soil: vermicompost }(1: 1) \text { v/v }+ \\
\text { mycorrhiza }\end{array}$ & 7.60 & 9.25 & 8.43 & 22.11 & 13.11 & 17.61 & 14.20 & 14.15 & 14.18 & 24.90 & 18.00 & 21.45 \\
\hline $\begin{array}{l}M_{4}-\text { soil: vermicompost: coco peat } \\
(1: 1: 1) \mathrm{v} / \mathrm{v}+\text { mycorrhiza }\end{array}$ & 7.40 & 11.45 & 9.43 & 31.08 & 27.99 & 29.54 & 19.85 & 14.00 & 16.93 & 34.70 & 17.30 & 26.00 \\
\hline $\begin{array}{l}M_{5}-\text { soil: vermicompost: rice husk } \\
(1: 1: 1) \text { v/v + mycorrhiza }\end{array}$ & 9.25 & 7.90 & 8.58 & 34.78 & 18.11 & 26.45 & 19.65 & 12.40 & 16.03 & 24.00 & 23.15 & 23.58 \\
\hline $\begin{array}{l}\mathrm{M}_{6} \text { - soil: vermicompost: coco peat: rice } \\
\text { husk }(1: 1: 1: 1) \mathrm{v} / \mathrm{v}+\text { mycorrhiza }\end{array}$ & 10.00 & 9.15 & 9.58 & 38.75 & 25.51 & 32.13 & 19.90 & 16.90 & 18.40 & 31.00 & 24.00 & 27.50 \\
\hline $\begin{array}{l}\mathrm{M}_{7} \text { - soil: vermicompost: saw dust }(1: 1: 1) \\
\text { v/v + mycorrhiza }\end{array}$ & 10.30 & 7.50 & 8.90 & 37.49 & 17.16 & 27.33 & 18.40 & 13.85 & 16.13 & 33.65 & 16.75 & 25.20 \\
\hline $\begin{array}{l}M_{8} \text { - soil: vermicompost: coco peat: saw } \\
\text { dust }(1: 1: 1: 1) \mathrm{v} / \mathrm{v}+\text { mycorrhiza }\end{array}$ & 13.00 & 8.30 & 10.65 & 33.01 & 33.11 & 33.06 & 20.50 & 24.25 & 22.38 & 25.00 & 32.15 & 28.58 \\
\hline Mean & 9.26 & 8.36 & 8.81 & 28.77 & 19.99 & 24.39 & 17.52 & 15.23 & 16.37 & 26.06 & 21.19 & 23.63 \\
\hline Factor & S Em \pm & $\mathrm{CD}$ at $5 \%$ & & $\begin{array}{l}\mathrm{S} \\
\mathrm{Em} \pm\end{array}$ & $\mathrm{CD}$ at $5 \%$ & & $\mathrm{~S} \mathrm{Em} \pm$ & $\mathrm{CD}$ at $5^{c}$ & & $\mathrm{~S}$ Em \pm & $\mathrm{CD}$ at 5 & \\
\hline$\overline{\text { Variety (V) }}$ & 0.21 & 0.62 & & 2.06 & 6.06 & & 0.54 & 1.58 & & 1.14 & 3.36 & \\
\hline Medium (M) & 0.21 & 0.63 & & 1.54 & 4.54 & & 0.59 & 1.74 & & 0.81 & 2.37 & \\
\hline $\mathbf{V} \times \mathbf{M}$ & 0.43 & 1.27 & & 2.35 & 6.91 & & 0.90 & 2.64 & & 1.61 & 4.74 & \\
\hline
\end{tabular}


Table.4 Fresh weight of roots per cutting $(\mathrm{g})$ as influenced by rooting media in marigold varieties

\begin{tabular}{|c|c|c|c|c|c|c|c|c|c|c|c|c|}
\hline \multirow[t]{3}{*}{ Variety Medium } & \multicolumn{12}{|c|}{ Fresh weight of roots per cutting (g) } \\
\hline & \multicolumn{3}{|c|}{ at Shifting } & \multicolumn{3}{|c|}{ at 30 DAS } & \multicolumn{3}{|c|}{ at 60 DAS } & \multicolumn{3}{|c|}{ at 90 DAS } \\
\hline & B M 1 & $\begin{array}{c}\text { B M } \\
2\end{array}$ & Mean & B M 1 & $\begin{array}{c}\text { B M } \\
2\end{array}$ & Mean & B M 1 & $\begin{array}{c}\mathbf{B} \mathbf{M} \\
\mathbf{2}\end{array}$ & Mean & B M 1 & $\begin{array}{c}\text { B M } \\
2\end{array}$ & Mean \\
\hline $\mathrm{M}_{1}-$ soil & 0.70 & 0.90 & 0.80 & 0.99 & 1.08 & 1.04 & 9.25 & 12.05 & 10.65 & 14.51 & 26.65 & 20.58 \\
\hline $\mathrm{M}_{2}$ - soil + mycorrhiza & 1.20 & 0.60 & 0.90 & 1.91 & 2.38 & 2.15 & 14.60 & 9.75 & 12.18 & 33.55 & 14.80 & 24.18 \\
\hline $\begin{array}{l}\mathrm{M}_{3} \text { - soil: vermicompost }(1: 1) \mathrm{v} / \mathrm{v}+ \\
\text { mycorrhiza }\end{array}$ & 1.20 & 0.70 & 0.95 & 2.49 & 2.49 & 2.50 & 15.95 & 11.45 & 13.70 & 28.05 & 23.20 & 25.63 \\
\hline $\begin{array}{l}M_{4}-\text { soil: vermicompost: coco peat } \\
(1: 1: 1) \mathrm{v} / \mathrm{v}+\text { mycorrhiza }\end{array}$ & 1.30 & 0.90 & 1.10 & 7.41 & 7.75 & 7.58 & 28.55 & 8.80 & 18.68 & 23.05 & 40.40 & 31.73 \\
\hline $\begin{array}{l}\text { M5- soil: vermicompost: rice husk } \\
(1: 1: 1) \mathrm{v} / \mathrm{v}+\text { mycorrhiza }\end{array}$ & 1.30 & 0.70 & 1.00 & 3.58 & 4.08 & 3.83 & 28.15 & 7.55 & 17.85 & 35.02 & 18.05 & 26.54 \\
\hline $\begin{array}{l}M_{6}-\text { soil: vermicompost: coco peat: } \\
\text { rice husk (1:1:1:1) v/v + mycorrhiza }\end{array}$ & 1.40 & 0.90 & 1.15 & 8.24 & 7.41 & 7.83 & 51.05 & 9.15 & 30.10 & 45.25 & 25.50 & 35.38 \\
\hline $\begin{array}{l}M_{7}-\text { soil: vermicompost: saw dust } \\
(1: 1: 1) \text { v/v + mycorrhiza }\end{array}$ & 1.50 & 0.60 & 1.05 & 4.74 & 4.81 & 4.78 & 29.90 & 7.40 & 18.65 & 37.55 & 21.00 & 29.28 \\
\hline $\begin{array}{l}M_{8} \text { - soil: vermicompost: coco peat: } \\
\text { saw dust }(1: 1: 1: 1) \text { v/v + mycorrhiza }\end{array}$ & 1.50 & 0.90 & 1.20 & 9.16 & 6.74 & 7.96 & 56.80 & 31.90 & 44.35 & 38.05 & 40.80 & 39.43 \\
\hline Mean & 1.26 & 0.77 & 1.02 & 4.81 & 4.59 & 4.71 & 29.28 & 12.26 & 20.77 & 31.87 & 26.30 & 29.09 \\
\hline Factor & $\begin{array}{l}\mathrm{S} \\
\mathrm{Em} \pm\end{array}$ & \multicolumn{2}{|c|}{$\mathrm{CD}$ at $5 \%$} & $\begin{array}{l}\mathrm{S} \\
\mathrm{Em} \pm\end{array}$ & \multicolumn{2}{|c|}{$\mathrm{CD}$ at $5 \%$} & $\begin{array}{l}\mathrm{S} \\
\mathrm{Em} \pm\end{array}$ & \multicolumn{2}{|c|}{$\mathrm{CD}$ at $5 \%$} & $\begin{array}{l}\mathrm{S} \\
\mathrm{Em} \pm\end{array}$ & \multicolumn{2}{|c|}{$\mathrm{CD}$ at $5 \%$} \\
\hline Variety (V) & 0.06 & \multicolumn{2}{|l|}{0.17} & 0.052 & \multicolumn{2}{|l|}{0.15} & 4.01 & \multicolumn{2}{|l|}{11.76} & 1.31 & \multicolumn{2}{|l|}{3.85} \\
\hline Medium (M) & 0.10 & \multicolumn{2}{|l|}{0.30} & 0.556 & \multicolumn{2}{|l|}{1.63} & 2.25 & \multicolumn{2}{|l|}{6.60} & 1.23 & \multicolumn{2}{|l|}{3.63} \\
\hline $\mathbf{V} \times \mathbf{M}$ & 0.13 & \multicolumn{2}{|l|}{0.40} & 0.68 & \multicolumn{2}{|l|}{2.00} & 3.89 & \multicolumn{2}{|l|}{11.43} & 2.43 & \multicolumn{2}{|l|}{7.15} \\
\hline
\end{tabular}


Table.5 Dry weight of roots (g) per cutting as influenced by rooting media among marigold varieties

\begin{tabular}{|c|c|c|c|c|c|c|c|c|c|c|c|c|}
\hline \multirow[t]{3}{*}{ Variety Medium } & \multicolumn{12}{|c|}{ Dry weight of roots per cutting (g) } \\
\hline & \multicolumn{3}{|c|}{ at Shifting } & \multicolumn{3}{|c|}{ at $30 \mathrm{DAS}$} & \multicolumn{3}{|c|}{ at 60 DAS } & \multicolumn{3}{|c|}{ at 90 DAS } \\
\hline & $\begin{array}{c}\text { B M } \\
1\end{array}$ & $\begin{array}{c}\text { B M } \\
2\end{array}$ & $\begin{array}{c}\text { Mea } \\
\mathbf{n}\end{array}$ & B M 1 & B M 2 & Mean & B M 1 & B M 2 & Mean & $\begin{array}{c}\text { B M } \\
1\end{array}$ & B M 2 & Mean \\
\hline $\mathbf{M}_{1}-$ soil & 0.17 & 0.15 & 0.16 & 0.25 & 0.29 & 0.28 & 5.25 & 3.70 & 4.48 & 11.31 & 7.25 & 9.28 \\
\hline $\mathbf{M}_{2}-$ soil + mycorrhiza & 0.19 & 0.14 & 0.17 & 0.74 & 0.64 & 0.69 & 5.75 & 5.47 & 5.61 & 11.52 & 12.10 & 11.81 \\
\hline $\begin{array}{l}\mathrm{M}_{3} \text { - soil: vermicompost }(1: 1) \mathrm{v} / \mathrm{v}+ \\
\text { mycorrhiza }\end{array}$ & 0.21 & 0.15 & 0.18 & 0.79 & 0.62 & 0.71 & 9.00 & 4.30 & 6.65 & 14.64 & 11.00 & 12.82 \\
\hline $\begin{array}{l}M_{4}-\text { soil: vermicompost: coco peat } \\
(1: 1: 1) \mathrm{v} / \mathrm{v}+\text { mycorrhiza }\end{array}$ & 0.24 & 0.21 & 0.23 & 1.82 & 0.85 & 1.34 & 12.00 & 3.30 & 7.65 & 19.54 & 12.60 & 16.07 \\
\hline $\begin{array}{l}M_{5} \text { - soil: vermicompost: rice husk } \\
(1: 1: 1) v / v+\text { mycorrhiza }\end{array}$ & 0.21 & 0.16 & 0.19 & 1.68 & 0.54 & 1.11 & 7.75 & 6.75 & 7.25 & 14.64 & 16.50 & 15.57 \\
\hline $\begin{array}{l}M_{6}-\text { soil: vermicompost: coco peat: rice } \\
\text { husk }(1: 1: 1: 1) \mathrm{v} / \mathrm{v}+\text { mycorrhiza }\end{array}$ & 0.26 & 0.25 & 0.26 & 1.73 & 1.73 & 1.73 & 13.75 & 6.55 & 10.15 & 23.54 & 12.00 & 17.77 \\
\hline $\begin{array}{l}M_{7}-\text { soil: vermicompost: saw dust } \\
(1: 1: 1) \mathrm{v} / \mathrm{v}+\text { mycorrhiza }\end{array}$ & 0.24 & 0.16 & 0.20 & 0.94 & 0.62 & 0.78 & 7.15 & 6.70 & 6.93 & 15.68 & 13.30 & 14.49 \\
\hline $\begin{array}{l}M_{8} \text { - soil: vermicompost: coco peat: } \\
\text { saw dust }(1: 1: 1: 1) \mathrm{v} / \mathrm{v}+\text { mycorrhiza }\end{array}$ & 0.21 & 0.24 & 0.23 & 2.29 & 0.58 & 1.44 & 4.50 & 11.30 & 7.90 & 23.54 & 11.80 & 17.67 \\
\hline Mean & 0.21 & 0.18 & 0.20 & 1.28 & 0.73 & 1.01 & 8.02 & 5.78 & 6.90 & 16.80 & 12.27 & 14.44 \\
\hline Factor & $\begin{array}{l}\mathrm{S} \\
\mathrm{Em} \pm\end{array}$ & $\mathrm{CD}$ at & & $\mathrm{S} \mathrm{Em} \pm$ & $\mathrm{CD}$ at 5 & & $\mathrm{~S} \mathrm{Em} \pm$ & $\mathrm{CD}$ at $5 \%$ & & $\begin{array}{l}\mathrm{S} \\
\mathrm{Em} \pm\end{array}$ & $\mathrm{CD}$ at 5 & \\
\hline Variety (V) & - & NS & & 0.12 & 0.37 & & 0.52 & 1.54 & & $1.1 \overline{1}$ & 3.27 & \\
\hline Medium (M) & 0.02 & 0.07 & & 0.09 & 0.28 & & 0.38 & 1.11 & & 0.59 & 1.74 & \\
\hline $\mathbf{V} \times \mathbf{M}$ & 0.02 & 0.07 & & 0.15 & 0.45 & & 0.79 & 2.32 & & 1.12 & 3.29 & \\
\hline
\end{tabular}


Table.6 Survival percentage of rooted cuttings $(\%)$ as influenced by rooting media among marigold varieties

\begin{tabular}{|c|c|c|c|}
\hline \multirow[t]{2}{*}{ Variety Medium } & \multicolumn{3}{|c|}{ Survival percentage of rooted cuttings $(\%)$} \\
\hline & B M 1 & B M 2 & Mean \\
\hline $\mathbf{M}_{1}-$ soil & 92.55 & 72.54 & 82.54 \\
\hline $\mathrm{M}_{2}-$ soil + mycorrhiza & 92.55 & 77.52 & 85.03 \\
\hline $\mathrm{M}_{3}$ - soil: vermicompost $(1: 1) \mathrm{v} / \mathrm{v}+$ mycorrhiza & 86.72 & 85.41 & 86.06 \\
\hline $\mathrm{M}_{4}-$ soil: vermicompost: coco peat $(1: 1: 1) \mathrm{v} / \mathrm{v}+$ mycorrhiza & 90.85 & 90.25 & 90.55 \\
\hline $\mathrm{M}_{5}$ - soil: vermicompost: rice husk $(1: 1: 1) \mathrm{v} / \mathrm{v}+$ mycorrhiza & 93.35 & 82.54 & 87.94 \\
\hline $\mathrm{M}_{6}-$ soil: vermicompost: coco peat: rice husk $(1: 1: 1: 1) \mathrm{v} / \mathrm{v}+$ mycorrhiza & 91.76 & 90.12 & 90.94 \\
\hline $\mathrm{M}_{7}$ - soil: vermicompost: saw dust $(1: 1: 1) \mathrm{v} / \mathrm{v}+$ mycorrhiza & 90.85 & 85.24 & 88.04 \\
\hline$M_{8}$ - soil: vermicompost: coco peat: saw dust $(1: 1: 1: 1) \mathrm{v} / \mathrm{v}+$ mycorrhiza & 95.71 & 94.85 & 95.78 \\
\hline Mean & 91.19 & 85.54 & 83.79 \\
\hline Factor & $\mathrm{S} \mathrm{Em} \pm$ & $\mathrm{CD}$ at $5 \%$ & \\
\hline Variety (V) & 0.73 & 2.12 & \\
\hline Medium (M) & 1.02 & 2.98 & \\
\hline$\overline{\mathbf{V X} \mathbf{M}}$ & 1.34 & 3.91 & \\
\hline
\end{tabular}


The maximum fresh weight of roots was recorded by the terminal cuttings planted in coco peat based media which might be due to better aeration and drainage conditions and water maintenance capability (Khayyat et al., 2007).

Presence of vermicompost acted as synergistic factor along with cocopeat, which might be due to the reason that vermicompost being a rich source of macro and micronutrients enhanced the availability of these nutrients in an easily available form.

Earthworm castings are known to be rich source of growth promoting substances viz., growth hormones, enzymes, antibiotics and vitamins (Bawalkar, 1992). The enriched food and nutrient status of the rooting media and consequent development of conducting tissues could have facilitated greater uptake of water and thus leading to a higher fresh weight of roots in mycorrhizal inoculated media combinations especially in cocopeat + vermicompost based mixtures which could have complemented each other. Similar observations were made in gladiolus by Meenakshi et al., (2015).

\section{Dry weight of roots per cutting (g) (Table 5)}

Among the rooting media, at 90 DAS the highest dry weight of roots (17.77 g) was recorded by M6- soil: vermicompost: coco peat: ricehusk $(1: 1: 1: 1) \mathrm{v} / \mathrm{v}+$ mycorrhiza which was on par with M8- soil: vermicompost: coco peat: sawdust (1:1:1:1) v/v + mycorrhiza (17.67 g) and M4 - soil: vermicompost: coco peat $(1: 1: 1) \mathrm{v} / \mathrm{v}+$ mycorrhiza (16.07 g) but significantly superior to M3 - soil: vermicompost (1:1) v/v + mycorrhiza (12.82 g). The lowest dry weight of roots $(9.28 \mathrm{~g})$ was recorded on M1 - soil. A high dry weight of roots might be due to a high value of corresponding fresh weight which might in turn due to a large number of sprouted roots that survived and grew longer up to 90 days after shifting.

Terminal cuttings planted in coco peat based media combinations recorded the maximum dry weight of roots which could be attributed to more number and length of roots. Bano et al., (1987) reported that vermicompost is a rich source of plant nutrients $(\mathrm{N}, \mathrm{P} 2 \mathrm{O} 5$, and $\mathrm{K} 2 \mathrm{O}$ ), secondary elements $(\mathrm{Ca}$ and $\mathrm{Mg}$ ) and vital micronutrients like $\mathrm{Fe}, \mathrm{B}, \mathrm{Zn}$ and $\mathrm{Mo}$, which directly or indirectly enhanced dry weight of roots (Meenakshi et al., 2015).

\section{Survival percentage of rooted cuttings (\%)} (Table 6)

Among the rooting media, the maximum survival of rooted cuttings $(95.78 \%)$ was recorded by M8 - soil: vermicompost: coco peat: saw dust $(1: 1: 1: 1) \mathrm{v} / \mathrm{v}+$ mycorrhiza on par with M6 - soil: vermicompost: coco peat: rice husk (1:1:1:1) v/v + mycorrhiza $(90.94 \%)$ and M4 - soil: vermicompost: coco peat $(1: 1: 1) \mathrm{v} / \mathrm{v}+$ mycorrhiza $(90.55 \%)$. The minimum percentage of rooted cuttings $(82.54 \%)$ was recorded on M1 - soil.

The cuttings planted in coco peat media combinations gave the highest survival percentage which might be due to its corresponding merit in root and shoot growth and sustenance over a period of time. The advantages with coco peat might be due to incorporation of coarser material that would improve the aeration status of medium (Awang et al., 2009). Aeration is necessary for the gaseous exchange between the soil and atmosphere to remove $\mathrm{CO}_{2}$ released by roots and microorganisms in the soil to external atmosphere and supply of $\mathrm{O}_{2}$ from the external atmosphere to the growing roots leading to better respiration and survival of plants (Jeyaseeli and Paul, 2010). Existence of vermicompost in rooting media could have 
enriched it with plant nutrients $\left(\mathrm{N}, \mathrm{P}_{2} \mathrm{O}_{5}\right.$, and $\mathrm{K}_{2} \mathrm{O}$ ), secondary elements $(\mathrm{Ca}$ and $\mathrm{Mg})$ and vital micronutrients like $\mathrm{Fe}, \mathrm{B}, \mathrm{Zn}$ and $\mathrm{Mo}$, having direct and indirect role in various metabolic activities. Further, the mycorhizal presence might have stimulated greater development of conducting tissues which facilitated more uptake of water and finally these facts might had led to a greater survival of rooted cuttings as also observed in gladiolus by Meenakshi et al., (2015). Existence of cocopeat + vermicompost was found to show synergistic and significant influence as compared to the other components like rice husk and saw dust because the treatments were found to be meritorious with the cocopeat + vermicompost only as compared to the other mixtures irrespective of whether the rice husk or saw dust were present or not.

\section{References}

Abdul, K, Muhammad, J, Bilquees, F. and Bushra, S. 2013. Clonal multiplication of guava through softwood cuttings under mist conditions. Pakistan Journal of Agricultural Sciences. 50(1): 23 - 27.

Awang, Y, Shaharom, A. S, Mohamad, R.B. and Selamat, A. 2009. Chemical and physical characteristics of coco peat based media mixtures and their effects on growth and development of Celosia cristata. American Journal of Agricultural and Biological Science. 4: $63-71$.

Bano, K, Kale R, and Gajanan, G. 1987. Culturing of earth worms Eudriluseugenia for cast production and assesment of worm cast as biofertilizer. Journal of Soil Biology and Ecology. 7: $98-105$.

Bawalkar. 1992. Vermicastings the effective biofertilizers. Kisan World. 7: 35 - 37.

Bharathy, P.V. Sonawane, P.C. and Sasnu, A. 2003. Effect of different planting media on rooting of cuttings in carnation (Dianthus caryophyllus L.). Journal of Maharastra Agricultural University. 28(3): $343-44$.

Davies, F.T. J. 1987. Effect of VAmycorrhizal fungi on growth and nutrient uptake of cuttings of Rosa multiflora in two container media with three level of fertilizers application. Plant and Soil. 104: 31 - 35.

Edwards, C. A. and Neuhauser, E.F. (1988). Earthworms in Waste and Environmental Management. Academic Publications. Co. The Hague Netherlands, pp 391.

Ellyard, R.K. and Ollerenshaw, P.J. 1984. Effect of indole butyric acid, medium composition, and cutting type on rooting of Grevillea johnsonii cuttings at two basal temperatures. Combined Proceedings of the International Plant Propagators Society. 34: $101-08$.

Ismail, S.M. and Asghar, H.I. 2007. Effect of indole butyric acid and types of cuttings on root initiation of Ficushawaii. Sarhad Journal of Agriculture. 23(4): $919-25$.

Jain, A.K, Dand, S.B. and Seng, K. 1999. Micropropagation through axillary bud multiplication in different mulberry genotype. Plant Cell Reports. 8: 737 40.

Jeyaseeli, D.M. and PaulRaj, S. 2010. Chemical characteristics of coir pith as a function of its particle size to be used as soilless medium. An International Quarterly Journal of Environmental Sciences. 4(2\&3): $163-69$.

Khayyat, M. Nazari, F. and Salehi, H. 2007 Effects of Different Pot Mixtures on Pothos (Epipremnumaureum Lindl. and Andre Golden Pothos) Growth and Development. American-Eurasian Journal of Agriculture and Environmental Sciences 2 (4): 341 - 48. 
Khewale, A. P, Golliwar, V. J, Poinkar, M. S, Jibhakate, S. B. and Athavale, M. P. 2005. Influence of different concentrations of IBA and media on root parameters in the propagation of carnation cv. Gaudina. Journal of Soils and Crops. 15(2): $406-10$.

Laubscher, C. P. (1990). Rooting techniques for select tree species. Unpublished Cape Technikon, Cape Town.

Lokesha, R, Mahishi, D.M. and Shivashankar, G. 1988. Studies on the use of coconut coir dust as a rooting media. Current Research. 17(12): 157 - 158.

Mahale, V.G. Ashok, T.H. and Kale, R.D. 2002. Vermicompost as rooting medium for carnation (Dianthus caryophyllus L.). Journal of Plant Biology. 29(2):175 -178 .

Malik, M.A, Muhammad, K.R, Muhammad, A.J, Saeed, A, Sitwat, R. and Javaid, I. 2013. Production of true-to-type guava nursery plants via application of IBA on soft wood cuttings. Journal of Agricultural Research. 51(3): $1-8$.

Mcgonigle, T. P. and Miller, M. H. 1996. Development of fungi below ground in association with plants growing in disturbed and undisturbed soils. Soil Biology and Biochemistry, 28: 263 269.

MeenakshiBasoli, Prabhat Kumar and Santosh Kumar. 2015. Impact of integrated nutrient management on growth and flowering of gladiolus (Gladiolus hybrida) cv. Novalux. Indian J. Agri. Sci., 85(5): 35-39.

Miller, M. H. 2000. Arbuscular mycorrhizae and the phosphorus nutrition of maize: A review of Guelph studies. Canadian Journal of Plant Pathological Science. 80: $47-52$.

Neelima, P, Singh, A. and Ahlawat, T.R. 2016. Influence of bio-fertilizers, chemicals and organic growth enhancers on growth and flower quality of gerbera under naturally ventilated polyhouse. Current Plant Science. 2(1): $39-43$.

Newton, A.C. Muthoka, P. and Dick, J.M. 1992. The influence of leaf area on the rooting physiology of leaf stem cuttings of Terminalia spinosa England. Tree structure and function. 6: 210 - 15.

Norman, Q. A. and Edward, C.V. 2005. Effect of vermicompost on plant growth. International symposium on vermin technologies for developing countries. Los Banos, Philippines. pp. $16-18$.

Ochoa, J, Banon, S, Fernandez, J. A, Franco, J. A. and Gonzalez, A. 2003. Influence of cutting position and rooting media on rhizogenesis in oleander cuttings. Acta Horticulture, 608: 101 - 06.

Panse, V.G. and Sukhatme. 1997. Statistical methods for Agricultural workers, ICAR, New Delhi. Pp - 381.

Rahman, H.U, Khan, M.A, Khokhar, K.M, Laghari, H.M and Rahman, H. 1991. Effect of season on rooting tip cuttings of guava treated with paclobutrazol. Indian Journal of Agriculture Science. 61: $404-06$.

Reddy, P. P. 2008. Organic Farming for Sustainable Horticulture. Scientific Publishers, Jodhpur, India. pp.20, 36, 66, 67.

Riaz, A, Khalil, U.R, Muhammad, I, Muhammad, I. and Muhammad, A.R. 2007.

Rubasinghe, M.K, Amarasinghe, K.G.K.D. and Krishnarajha, S.A. 2009. Effect of rooting media, naphtheline acetic acid (NAA) and gibberelic acid (GA3) on growth performances of Chiritamoonii. Ceylon Journal of Science, Biological Sciences. 38(1): 17 - 22.

Shirol, A.M, Kulkarni, B.S, Reddy, B.S, Kanamadi, V.C. and Thammaih, N. 2001. Influence of different rooting media on rootability of tip cuttings of 
dwarf poinsettia. Karnataka Journal of Agricultural Sciences. 14(4): 1145 - 46.

Siddagangaiah, Vadiraj, B.A, Sudharshan,

M.R. and Krishnakumar, V. 1996.

Standardisation of rooting media for propagation of vanilla (Vanilla planifolia Andr.). Journal of Spices and Aromatic Crops. 5 (2): 131 - 133.

Singh, K.P. Suchitra, Raghava, S.P.S. and Misra, R.L. 2002 Effect of media on rooting of carnation cuttings. Journal of Ornamental Horticulture 5(2): 53.
Smith, Ch. 1995. Coir: a viable alternative to peat for potting. The Horticulturist. 4(3): $12-25$.

Smith, S.E. and Read, D.J. 2008. Mycorrhizal symbiosis. 3rd edition. Academic press, London, U K.

Wahab, F, Nabi, G, Ali, N. and Shah, M. 2001. Rooting response of semi hard wood cuttings of guava (Psidium guajava L.) to various concentrations of different auxins. Online Journal of Biological Sciences. 1(4): 184-87.

\section{How to cite this article:}

Vijay, J., A.V.D. Dorajeerao, K. Umajyothi and Salomi Suneetha, D.R. 2018. Effect of Media on Root Parameters in Marigold. Int.J.Curr.Microbiol.App.Sci. 7(11): 3388-3401. doi: https://doi.org/10.20546/ijcmas.2018.711.389 